\title{
Analysis of microbiota in elderly patients with Acute Cerebral Infarction
}

\author{
Lin Huang ${ }^{1}$, Teng Wang ${ }^{1}$, Qian Wu ${ }^{1}$, Xin Dong ${ }^{1}$, Feifei Shen ${ }^{1}$, Dong Liu ${ }^{1}$, Xiaoxuan Qin ${ }^{1}$, Lanyun Yan ${ }^{\text {Corresp., } 1}$, Qi \\ Wan ${ }^{\text {Corresp. } 1}$ \\ ${ }^{1}$ Department of Neurology, The First Affiliated Hospital of Nanjing Medical University, Nanjing, Jiangsu Province, China \\ Corresponding Authors: Lanyun Yan, Qi Wan \\ Email address: yanlanyun@126.com, wanqi@jsph.org.cn
}

\section{Abstract}

Background and Aims: Recent evidence suggest that microbiota is associated with almost all major types of diseases, including cardiovascular diseases. However, its role in Acute Cerebral Infarction remains unexplored. It is important to understand the diversity and distribution of gut microbiota (GM) in patients with Acute Cerebral Infarction and the role that GM plays in this type of disease.

Methods: We performed pyrosequencing on the gut microbiota of 40 individuals in order to elucidate whether the composition of the microbiota differs between patients with Acute Cerebral Infarction and healthy controls: Of these individuals, there were 31 with Acute Cerebral Infarction and 9 controls. We applied linear regression to calculate the correlation between the gut flora and disease risk factors. Finally, KEGG functional enrichment analysis was conducted to examine the correlation between the gut flora and Acute Cerebral Infarction.

Results: The overall microbial structure was similar in both the controls and the patients, but the control group had higher relative presence of Blautia obeum while the presence of Streptococcus infantis and Prevotella copri were relatively higher in the patient group. Using linear regression, we found that Blautia obeum was negatively associated with white blood cell count and Streptococcus infantis was positively correlated with creatinine and lipoprotein. The KEGG pathway analysis indicated that the bio-pathways including methane metabolism, lipopolysaccharide synthesis, bacterial secretion, and flagellar assembly of the gut microbiota in the patient group was expressed differently than that of the controls. We identified three differentially expressed gut microbial functions in Acute Cerebral Infarction and found four bacterial pathways that might be related to the development of this disease.

Conclusions : Our study identified three abnormally-expressed bacteria --, Blautia obeum, Streptococcus infantis, and Prevotella copri -- in patients with Acute Cerebral Infarction 
compared with healthy controls. It reveals a correlation of these bacterial species with Acute Cerebral Infarction as they relate to disease factors and functional pathways. These findings may shed light on the treatment of cerebral i nfarction because gut microbiota could serve as a potential therapeutic approach for the treatment of cardiovascular and metabolic diseases. 
2 Lin Huang, Teng Wang, Qian Wu, Xin Dong, Feifei Shen, Dong Liu, Xiaoxuan Qin, Lanyun Yan *, Qi

3 Wan *

4 From Department of Neurology, The First Affiliated Hospital of Nanjing Medical University, 300

5 Guangzhou Road, Nanjing, Jiangsu Province, China.

6

*Correspondences:

17 Qi Wan, MD, E-mail: qi_wan@126.com;

18 Lanyun Yan, MD, E-mail: yanlanyun@126.com 
Abstract

Background and Aims: Recent evidence suggest that microbiota is associated with almost all major types of diseases, including cardiovascular diseases. However, its role in Acute Cerebral Infarction remains unexplored. It is important to understand the diversity and distribution of gut microbiota (GM) in patients with Acute Cerebral Infarction and the role that GM plays in this type of disease.

Methods: We performed pyrosequencing on the gut microbiota of 40 individuals in order to elucidate whether the composition of the microbiota differs between patients with Acute Cerebral Infarction and healthy controls: Of these individuals, there were 31 with Acute Cerebral Infarction and 9 controls. We applied linear regression to calculate the correlation between the gut flora and disease risk factors. Finally, KEGG functional enrichment analysis was conducted to examine the correlation between the gut flora and Acute Cerebral Infarction.

Results: The overall microbial structure was similar in both the controls and the patients, but the control group had higher relative presence of Blautia obeum while the presence of Streptococcus infantis and Prevotella copri were relatively higher in the patient group. Using linear regression, we found that Blautia obeum was negatively associated with white blood cell count and Streptococcus infantis was positively correlated with creatinine and lipoprotein. The KEGG pathway analysis indicated that the bio-pathways including methane metabolism, lipopolysaccharide synthesis, bacterial secretion, and flagellar assembly of the gut microbiota in the patient group was expressed differently than that of the controls. We identified three differentially expressed gut microbial functions in Acute Cerebral Infarction and found four bacterial pathways that might be related to the development of this disease.

Conclusions: Our study identified three abnormally-expressed bacteria --, Blautia obeum, Streptococcus infantis, and Prevotella copri -- in patients with Acute Cerebral Infarction compared with healthy controls. It reveals a correlation of these bacterial species with Acute Cerebral Infarction as they relate to disease factors and functional pathways. These findings may shed light on the treatment of cerebral infarction because gut microbiota could serve as a potential therapeutic approach for the treatment of cardiovascular and metabolic diseases.

\section{Introduction}

Stroke causes 6.2 million deaths globally (Esenwa \& Gutierrez 2015), making it the second leading cause of death. Cerebral infarction, also known as cerebral stroke, accounts for 87 percent of all stroke cases (Roger et al. 2011), and occurs when the arteries that supply blood to the brain are blocked or narrowed so 
62 the development of fatty deposits in the lining of the vessel walls. Tissue plasminogen activator (TPA) 63 (Raphaeli et al. 2015) is a common treatment for cerebral infarction, but many patients do not arrive at the 64 hospital within the limited time needed to effectively access brain-saving treatments. Thus it is critical to 65 identify a stroke and to seek treatment immediately to have the best possible chance of a full recovery. Dysbiosis of the gut microbiota has not only been shown to contribute to the development of the immune system (Butto \& Haller 2016), but also, remarkably, to the development of the Central Nervous System (CNS) (Borre et al. 2014). Gut microbiota is related to the main systems of the human body, such as the immune system, nervous system, endocrine system, digestive system, respiratory system and even the circulatory system. Atherosclerosis and stroke are two major diseases of the circulatory system. The major culprit in these diseases is trimethylamine oxide (TMAO) (Wu et al. 2018). The formation of TMAO is closely related to the metabolism of intestinal flora. This information leads to the question of whether the gut microbiota in patients with Acute Cerebral Infarction is different from those without cerebral infarction. Furthermore, it is possible that different microbiota might be further related to other indicators of disease. The above hypothesis can further elaborate the theory of a brain-gut axis and may provide a theoretical basis for the prevention and treatment of Acute Cerebral Infarction. Researchers have identified variation in the composition of gut microbiota in stroke patients (Houlden et al. 2016; Singh et al. 2016; Yin et al. 2015) but only a few experimental studies have been published which focus on the role of gut microbiota in cerebral infarction. The link between gut flora and Acute Cerebral Infarction remains to be established, so the exact mechanisms of how gut microbiota are involved in cerebral infarction are yet to be determined.

To elucidate this relationship, we conducted the described study in which 40 samples (31 from patients with Acute Cerebral Infarction and nine from controls) were analyzed for 16S rRNA genes by pyrosequencing to detect differences in the microbial composition of the gut and to explore the correlation between gut flora and Acute Cerebral Infarction using linear regression and KEGG enrichment analysis.

\section{Methods and Results}

Methods

\section{Sample collection}

Fecal samples were collected from patients, whose median age was 61 years old, with Acute Cerebral Infarction from the First Affiliated Hospital of Nanjing Medical University, from February 2018 to May 2018. There was no negative control for age or gender groups; instead, the range was set from 40 to 80 years. The main inclusion criteria for the study group was Acute Cerebral Infarction as diagnosed by a neurologist. All patients underwent Magnetic Resonance Imaging (MRI) or a Computed Tomography (CT) scan to assess ischemic lesions. The large-artery atherosclerotic subtype of atherosclerosis was determined according to the TOAST classification system. Radiological evidence of intracerebral hemorrhage, a 
programmed stroke, cardiac stroke, or other TOAST subtypes), serious comorbidity, or a medical condition within one month prior to admission (i.e., patients with congestive heart failure, respiratory failure, renal failure, or severe liver dysfunction), or those taking probiotics or antibiotics, were excluded from the study group. The control group consisted of asymptomatic participants (according to a physical examination and self-report of no acute disease). The control group excluded participants who had taken antibiotics or probiotics within a month prior to admission, or who had a history of cerebrovascular disease. In addition, we excluded interference from diabetes, coronary heart disease, and renal dysfunction. Color Doppler imaging and transcranial Doppler ultrasound were performed in all control groups to determine cardiovascular status. The trial and informed consent was approved by the ethics committee of the First Affiliated Hospital of Nanjing Medical University (2018-SRFA-122) (In supplement).

Fresh stool samples were collected in the morning to avoid surface contamination and urine contamination. Internal stool samples of 3-5 g were collected with a clean sterile spoon and were placed in a sterile, airtight tube (QIAGEN). The sample was preserved immediately in an anaerobic environment in a liquid nitrogen tube in order to avoid repeated freezing and thawing. After transportation on dry ice, the sample was stored at $-80{ }^{\circ} \mathrm{C}$. The fecal stool collection in the patient group was completed within 48 hours of admission.

\section{Detection of biochemical specimens}

All fasting venous blood samples of about $5 \mathrm{~mL}$ were collected into the drying tube and then shot through the barrel to the pretreatment room. After the barcode was brushed in the pretreatment room, the samples were put into the Beckman Coulter assembly line. On the assembly line, each specimen was centrifuged for 7 minutes at 3000 rotations. The centrifuged specimen was detected by the Beckman Coulter AU5800 biochemical analyzer (Beckman Coulter, Inc, Brea, CA, USA). Different testing reagents were used according to the different samples being tested (the reagents needed for related testing items are in Table S1).

\section{Routine blood test}

The $2 \mathrm{~mL}$ venous blood samples, which were fasting samples taken early in the morning, were collected into the EDTA anticoagulant tube without centrifugation and entered the Beckman Coulter pipeline (Unicel DxH 800).

\section{PCR amplification and pyrosequencing of bacterial 16S rRNA genes}

Bacterial DNA was extracted from fecal samples according to the manufacturer's instructions using the Fecal DNA Extraction Kit (QIAGEN). The V4 variable region of the bacterial 16S rRNA gene was amplified by polymerase chain reaction (PCR) using the bar code primers 514F (GTGCCAGCMGCCGCGTAA) and 805R (GGACTACHVGGGTWTCTAAT). The PCR cycle conditions were: initial denaturation at $94{ }^{\circ} \mathrm{C}$ for two minutes; denaturation at $94{ }^{\circ} \mathrm{C}$ for 30 seconds; denaturation at $52{ }^{\circ} \mathrm{C}$ for 30 seconds; denaturation at $72{ }^{\circ} \mathrm{C}$ for 45 seconds; and extension at $72{ }^{\circ} \mathrm{C}$ for five minutes. Each $25 \mu \mathrm{L}$ reaction consisted of $0.5 \mu \mathrm{L}$ of template DNA, $2.0 \mu \mathrm{L}$ of dNTP mix $(2.5 \mathrm{mmol} / \mathrm{L}$; 
132 Takara), $2.5 \mu \mathrm{L}$ of Takara $10 \mathrm{Ex}$ Taq buffer $\left(\mathrm{Mg}^{+}\right.$free $), 1.5 \mu \mathrm{L}$ of $\mathrm{Mg}^{+}(25 \mathrm{mmol} / \mathrm{L}), 0.25 \mu \mathrm{L}$ of Takara

133 Ex Taq DNA polymerase (2.5 units), $0.5 \mu \mathrm{L}$ of $10 \mu \mathrm{mol} / \mathrm{L}$ bar code primer $514 \mathrm{~F}, 0.5 \mu \mathrm{L}$ of $10 \mu \mathrm{mol} / \mathrm{L}$

134 primer $805 \mathrm{R}$, and $17.25 \mu \mathrm{L}$ of double-distilled water. All dilution was carried out with sterile double 135 distilled water.

136 All PCR products were combined and sent to Beijing Genomic Institute for sequencing using the Illumina 137 Miseq (PE 150), according to the manufacturer's protocol (Zhou et al. 2011).

138 5. Statistical analysis

139 The tag number for each taxonomic rank (species) or OUT (operational taxonomic units) in the samples

140 was summarized in a profiling table or histogram, drawn with R (v3.1.1) software. The nonparametric Wilcoxon Rank-Sum test was used to identify OTU among different groups in QIIME. In order to determine the differences between the two groups not reported in QIIME, the OTUs of $\mathrm{P}<0.1$ in QIIME analysis were classified into generic levels and then statistical tests were implemented using Graphpad prim6. A chisquare test was used for categorical variables using SPSS. A value of $\mathrm{P}<0.05$ was considered statistically significant in the comparison groups.

\section{Results}

\section{Composition of gut flora in patients with Acute Cerebral Infarction and in controls}

In this study, a total of 40 blood and fecal samples were collected from 31 patients and nine healthy controls. Clinical characteristics of the whole population are shown in Table 1. The rates of previous HBP $(\mathrm{p}=0.005)$ and previous HLP $(p=0.036)$, as well as the levels of TC $(p=0.028)$, HDL $(p=0.002)$, TG $(p=0.003)$, WBC $(\mathrm{p}=0.023)$, and LP $(\mathrm{a})(\mathrm{p}=0.003)$ were significantly higher in the patients with Acute Cerebral Infarction than in the control subjects. In addition, there was no difference in GLU ( $p=0.178), \mathrm{Cr}(\mathrm{p}=$ $0.13)$ and UA $(p=0.134)$ between the two groups, which indicated that the patients sampled were significantly free of diabetes and renal dysfunction Table2.

To investigate whether gut microbiota composition differed between the study population of patients with cerebral infarction and healthy controls, we performed sequencing of the V4 region of the 16S ribosomal RNA gene from fecal samples. After filtering for quality, a total of 1,670,914 reads were included for downstream analysis and an average of $41,772 \pm 293$ sequences (SD) were assigned to each sample.

PLS-DA (Partial least squares discrimination analysis) was performed in order to further distinguish between the groups. This was achieved by rotating PCA (Principal Component Analysis) components such that a maximum separation between classes was obtained and to understand which variables carried the class separating information. The PCA components were calculated to analyze the composition of the gut microbiota in patients with Acute Cerebral Infarction and in the control group (Figure 1A). However, the diversity of species richness (represented by Chao, observed species, ace), richness and evenness (represented by Shannon and Simpson indexes) of the microbial community indicated, and the number of 
168

169

170

171

172

173

174

175

176

177

178

179

180

181

182

community species (Good's coverage) revealed that no significant difference in gut flora diversity could be identified between these two groups (Figure 1B-G).

\section{Gut bacterial species variations in Acute Cerebral Infarction patients and controls}

In contrast, the different taxa abundance (Class, Family, Genus, Order, Phylum and Species) (Figure S1-5 and Figure 2A) in the gut microbiota showed significant differences at the species level between patients with Acute Cerebral Infarction and the healthy controls. The bacterial species with the highest relative abundances were Blautia obeum $(\mathrm{p}=0.0441)$, Streptococcus infantis $(\mathrm{p}=0.017)$, and Prevotella copri $(\mathrm{p}$ $=0.0099$ ). The most remarkable difference was found in Blautia obeum. The presence of Blautia obeum is relatively lower in the patient group while Streptococcus infantis and Prevotella copri are relatively higher in cerebral infarction patients compared to the control group (Figure 2B-D).

\section{Correlation between gut microbial taxa at the species level and Acute Cerebral Infarction}

After identifying three abnormally-expressed gut microbiota species, we analyzed whether there were any associations between bacteria and cardiovascular risk factors using linear regression. Findings are indicated in Table 2. They show that Blautia was negatively associated with the white blood cell count $\left(\mathrm{r}^{2}=0.1053\right)$. In contrast, Streptococcus showed a positive correlation with creatinine $\left(\mathrm{r}^{2}=0.1328\right)$ and lipoprotein $\left(\mathrm{r}^{2}=\right.$ 0.1004), whereas Prevotella had no significant relation to any disease risk factors.

To predict the abundance of gene families and the related functional pathways of microbial communities in the fecal contents, KEGG functional pathway analysis, a predictive metabolism approach, was performed based on the 16S rRNA gene sequencing and Green Genes database (Figure 3). Results suggested that many bacterial pathways involved in methane $(\mathrm{p}=0.0103)$ (Figure $3 \mathrm{~B})$, lipopolysaccharide $(\mathrm{p}=0.0166$ and $\mathrm{p}=$ $0.0249)$ (Figure $3 \mathrm{C}$ and $\mathrm{F}$ ), secretion $(\mathrm{p}=0.0156)$ (Figure $3 \mathrm{D})$ and flagella $(\mathrm{p}=0.0065)$ (Figure $3 \mathrm{E}$ ) functions were significantly modulated in Acute Cerebral Infarction.

Functional analysis showed that the methane metabolism of these three bacteria was present in patients with Acute Cerebral Infarction. But lipopolysaccharide synthesis was enhanced in patients with Acute Cerebral Infarction. In addition, acute cerebral infarction may be positively correlated with bacterial secretion and flagellar assembly in these three gut microbiota.

\section{Discussion}

Our results support the concept of bidirectional communication along the brain-gut axis. Recent reports have shown that microbial populations play a significant role in development of cerebral infarction (Cryan \& Dinan 2012; Yin et al. 2015), and that diseases caused by antibiotic treatment can affect the outcome of stroke (Benakis et al. 2016). At present, bacteria have been considered as a pathogenic factor in the development of cardiovascular diseases. The difference of intestinal flora between atherosclerotic patients 
and unaffected people suggests that the change of intestinal flora may be related to atherosclerosis and that intestinal flora may change the metabolites of some substances, such as, TMAO (Yin et al. 2015). Studies have shown that increased levels of TMAO are associated with an increased risk for major cardiovascular and cerebrovascular incidents. TMAO can be used as an accurate screening tool for predicting the future risk for heart attack, stroke, and death among people not recognized by traditional risk factors and blood tests (Table1 and 2). Here, we report that Acute Cerebral Infarction itself significantly affects the intestinal microbial composition and that these changes may be associated with the occurrence and development of Acute Cerebral Infarction.

The intestinal microbiome has been further implicated in the pathogenesis of multiple diseases such as obesity, depressive disorders, chronic ileal inflammation, liver disease, and atherosclerosis (Chang et al. 2015; Fei \& Zhao 2013; Koeth et al. 2013; Le Roy et al. 2013; Llopis et al. 2016; Schaubeck et al. 2016; Zheng et al. 2016). For example, the metabolism of dietary L-carnitine, a nutrient in red meat, by intestinal microbiota was demonstrated to promote atherosclerosis and lead to cardiovascular disease risk by producing trimethylamine and trimethylamine-N-oxide (Koeth et al. 2013). However, the role of the intestinal microbiota in cerebral infarction, the development of diabetes, renal dysfunction, and other related diseases is not well understood ( $\mathrm{Ji}$ et al. 2017).

To address this void, the present study sought to determine whether the composition of the gut microbial community differed between Acute Cerebral Infarction patients and healthy controls, and whether the microbial taxa of the gut correlates with risk factors for cerebral infarction. In terms of microbiota composition, no significant difference was identified between the patient group and the control group (Figure 1). However, the control group showed a higher prevalence of Blautia obeum $(0.315 \%)$ in the gastrointestinal system than the disease group (0.115\%) (Figure 2).

Blautia obeum is a species of anaerobic, gram-positive bacteria found in the gut and is recognized as being dominant in the human colon (Lawson \& Finegold 2015). It has been reported that B. obeum, along with other relevant taxa, play an important role both in the recovery process from $V$. cholerae infection and the maturation of microbiota in children (Hsiao et al. 2014). There has been speculation about the possibility that some of these bacteria may be helpful in the repair of gut microbiota in individuals whose gut communities have been wounded through a variety of insults, including enteropathogen infections (Hsiao et al. 2014). In addition, D. Hatziioanou et. al. identified and characterized a gene cluster from the human gut isolate Blautia obeum A2-162 which encodes the novel nisin-like peptides NsoA1-3 and NsoA4. Moreover, the antimicrobial activity of the host strain could be detected in the presence of trypsin (Hatziioanou et al. 2017). Thus, based on our discovery, B. obeum may function as an anti-stroke factor and therefore its role in Acute Cerebral Infarction deserves a thorough investigation.

We also found that both Streptococcus infantis and Prevotella copri were expressed in greater quantity in Acute Cerebral Infarction patients than in healthy people. Streptococcus infantis is reported to cause minocycline resistance; its genetic basis is due to tet (S) present on a novel low copy number plasmid flanked by IS 1216 elements, which likely mediate its excision (Ciric et al. 2014). Prevotella are members 
237

238

239

240

241

242

243

244

245

246

247

248

249

250

251

252

253

254

255

256

257

258

259

260

261

262

263

264

265

266

267

268

269

270

271

272

of the oral, vaginal, and gut microbiota and are often recovered from anaerobic infections of the respiratory tract. Overgrowth of Prevotella in other diseases is known to occur in cases, such as in hypertension (Li et al. 2017) and chronic inflammatory disease (Larsen 2017). When compared with strict commensal bacteria, Prevotella exhibits increased inflammatory properties and studies indicate that Prevotella predominantly activates the toll-like receptor 2, leading to the production of Th17-polarizing cytokines by antigen presenting cells, including interleukin-23 (IL-23) and IL-1. Furthermore, the expansion of Prevotella copri is associated with enhanced susceptibility to arthritis, and the colonization of mice with the bacteria revealed the ability of $P$. copri to dominate the intestinal microbiota, resulting in an increased sensitivity to chemically induced colitis (Scher et al. 2013). There is growing evidence linking Prevotella copri to human diseases (Scher et al. 2013). Our study revealed that these two bacteria were found in relatively higher abundance in those with Acute Cerebral Infarction than in unaffected patients; whether they exert an indirect influence on the pathogenesis of this disease remains to be determined.

The correlation between gut flora and disease risk factors was also analyzed using linear regression. The data revealed a negative correlation between Blautia's and WBC $\left(\mathrm{r}^{2}=0.1053, \mathrm{P}=0.04\right)$, and a positive correlation between Streptococcus, creatinine $\left(\mathrm{r}^{2}=0.1328, \mathrm{P}=0.022\right)$ and lipoprotein $\left(\mathrm{r}^{2}=0.1004\right.$, $\mathrm{P}=0.0494$ ) (Table3). Combined with previous findings, it may be concluded that Blautia may support the immune system, while Streptococcus may contribute to the development of Acute Cerebral Infarction.

There are reports (Brzosko et al. 2009; Mekhlafi et al. 2018) that impaired renal function is a strong risk factor for cardiovascular disease with a poor prognosis for the affected patients. Renal insufficiency can predict the long-term mortality after Acute Cerebral Infarction. However, the in-hospital mortality after Acute Cerebral Infarction is also closely related to the disturbance of consciousness, the severity of the cerebral infarction, body temperature, blood sugar, and other complications. $\mathrm{Cr}$ and UA are the main indicators of renal function. Although there is not much difference between the normal group and the disease group, there is a positive correlation between serum $\mathrm{Cr}$ and Streptococcus in the disease group, so it is speculated that Streptococcus may affect the kidney, which further influences the development of cardiovascular disease.

According to the report, we found that the key residues of lipoprotein PiaA, trp158, stabilized the ironchromium complex in Streptococcus pneumonia (Zhang et al. 2017). There is a need for further study of the idea that Streptococcus may form a complex with the lipoprotein and consequently promote the increase in the lipoprotein.

Finally, we compared the differences of microbial bio-pathway enrichment between the patient group and control group using KEGG enrichment analysis. The analysis revealed that methane metabolism was inhibited in patients with Acute Cerebral Infarction, while lipopolysaccharide synthesis $(p=0.0166$ and $p$ $=0.0249)$, secretion pathways $(p=0.0156)$ and the flagellar assembly process $(p=0.0065)$ were enhanced in these patients (Figure3).

In addition, due to the limitation of the sample size, the correlation of the results may not be particularly 
273

274

275

276

277

278

279

280

281

282

283

284

285

286

287

288

289

290

291

292

293

294

295

296

297

298

299

300

301

302

303

304

305

306

307

308

309

310

strong, so increasing the sample size to determine the accuracy of the results of this experiment and later clinical guidance ought to be completed. The metabolites of bacteria have not been tested, but a series of deviations caused by bacterial changes can only be predicted by bioinformatics. In subsequent experiments, we need to further explore the changes of microbial metabolites such as TMAO, short-chain fatty acids, and so on, in order to confirm the pathway of microbial changes affecting cerebral infarction.

In conclusion, this study identified three abnormally-expressed bacteria, Blautia obeum $(\mathrm{p}=0.0441)$, Streptococcus $(\mathrm{p}=0.017)$ and Prevotella $(\mathrm{p}=0.0099)$ in patients with Acute Cerebral Infarction compared with healthy controls. It also revealed a correlation of these bacterial species to Acute Cerebral Infarction as they relate to disease factors and functional pathways. These findings may shed light on the treatment of Acute Cerebral Infarction because gut microbiota could serve as a potential therapeutic approach for the treatment of cerebrovascular and metabolic diseases.

\section{References:}

Benakis C, Brea D, Caballero S, Faraco G, Moore J, Murphy M, Sita G, Racchumi G, Ling L, Pamer EG, Iadecola C, and Anrather J. 2016. Commensal microbiota affects ischemic stroke outcome by regulating intestinal gammadelta T cells. Nat Med 22:516-523. DOI 10.1038/nm.4068

Borre YE, O'Keeffe GW, Clarke G, Stanton C, Dinan TG, and Cryan JF. 2014. Microbiota and neurodevelopmental windows: implications for brain disorders. Trends Mol Med 20:509-518. DOI 10.1016/j.molmed.2014.05.002

Brzosko S, Szkolka T, and Mysliwiec M. 2009. Kidney disease is a negative predictor of 30-day survival after acute ischaemic stroke. Nephron Clin Pract 112:c79-85.

DOI 10.1159/000213085

Butto LF, and Haller D. 2016. Dysbiosis in intestinal inflammation: Cause or consequence. Int J Med Microbiol 306:302-309. DOI 10.1016/j.jimm.2016.02.010

Chang CJ, Lin CS, Lu CC, Martel J, Ko YF, Ojcius DM, Tseng SF, Wu TR, Chen YY, Young JD, and Lai HC. 2015. Ganoderma lucidum reduces obesity in mice by modulating the composition of the gut microbiota. Nat Commun 6:7489. DOI 10.1038/ncomms8489

Ciric L, Brouwer MSM, Mullany P, and Roberts AP. 2014. Minocycline resistance in an oral Streptococcus infantis isolate is encoded by tet( $\mathrm{S}$ ) on a novel small, low copy number plasmid. Fems Microbiology Letters 353:106115. DOI 10.1111/1574-6968.12410

Cryan JF, and Dinan TG. 2012. Mind-altering microorganisms: the impact of the gut microbiota on brain and behaviour. Nat Rev Neurosci 13:701-712. DOI 10.1038/nrn3346

Esenwa C, and Gutierrez J. 2015. Secondary stroke prevention: challenges and solutions. Vasc Health Risk Manag 11:437-450. DOI 10.2147/VHRM.S63791

Fei N, and Zhao L. 2013. An opportunistic pathogen isolated from the gut of an obese human causes obesity in germfree mice. ISME J 7:880-884. DOI 10.1038/ismej.2012.153 
Hatziioanou D, Gherghisan-Filip C, Saalbach G, Horn N, Wegmann U, Duncan SH, Flint HJ, Mayer MJ, and Narbad A. 2017. DOI Discovery of a novel lantibiotic nisin O from Blautia obeum A2-162, isolated from the human gastrointestinal tract. Microbiology-Sgm 163:1292-1305. DOI 10.1099/mic.0.000515

Houlden A, Goldrick M, Brough D, Vizi ES, Lenart N, Martinecz B, Roberts IS, and Denes A. 2016. Brain injury induces specific changes in the caecal microbiota of mice via altered autonomic activity and mucoprotein production. Brain Behav Immun 57:10-20.

DOI 10.1016/j.bbi.2016.04.003

Hsiao A, Ahmed AMS, Subramanian S, Griffin NW, Drewry LL, Petri WA, Haque R, Ahmed T, and Gordon JI. 2014. Members of the human gut microbiota involved in recovery from Vibrio cholerae infection. Nature 515:423-+. DOI 10.1038/nature13738

Ji WZ, Zhu Y, Kan PC, Cai Y, Wang ZD, Wu ZJ, and Yang P. 2017. Analysis of intestinal microbial communities of cerebral infarction and ischemia patients based on high throughput sequencing technology and glucose and lipid metabolism. Molecular Medicine Reports 16:5413-5417. DOI 10.3892/mmr.2017.7227

Koeth RA, Wang ZE, Levison BS, Buffa JA, Org E, Sheehy BT, Britt EB, Fu XM, Wu YP, Li L, Smith JD, DiDonato JA, Chen J, Li HZ, Wu GD, Lewis JD, Warrier M, Brown JM, Krauss RM, Tang WHW, Bushman FD, Lusis AJ, and Hazen SL. 2013. Intestinal microbiota metabolism of L-carnitine, a nutrient in red meat, promotes atherosclerosis. Nat Med 19:576-585. DOI 10.1038/nm.3145

Larsen JM. 2017. The immune response to Prevotella bacteria in chronic inflammatory disease. Immunology 151:363-374. DOI 10.1111/imm.12760

Lawson PA, and Finegold SM. 2015. Reclassification of Ruminococcus obeum as Blautia obeum comb. nov. International Journal of Systematic and Evolutionary Microbiology 65:789-793. DOI 10.1099/ijs.0.000015

Le Roy T, Llopis M, Lepage P, Bruneau A, Rabot S, Bevilacqua C, Martin P, Philippe C, Walker F, Bado A, Perlemuter G, Cassard-Doulcier AM, and Gerard P. 2013. Intestinal microbiota determines development of non-alcoholic fatty liver disease in mice. Gut 62:1787-1794. DOI 10.1136/gutjnl-2012-303816

Li J, Zhao FQ, Wang YD, Chen JR, Tao JE, Tian G, Wu SL, Liu WB, Cui QH, Geng B, Zhang WL, Weldon R, Auguste K, Yang L, Liu XY, Chen L, Yang XC, Zhu BL, and Cai J. 2017. Gut microbiota dysbiosis contributes to the development of hypertension. Microbiome 5. ARTN 14. DOI 10.1186/s40168-016-0222$\mathrm{x}$

Llopis M, Cassard AM, Wrzosek L, Boschat L, Bruneau A, Ferrere G, Puchois V, Martin JC, Lepage P, Le Roy T, Lefevre L, Langelier B, Cailleux F, Gonzalez-Castro AM, Rabot S, Gaudin F, Agostini H, Prevot S, Berrebi D, Ciocan D, Jousse C, Naveau S, Gerard P, and Perlemuter G. 2016. Intestinal microbiota contributes to individual susceptibility to alcoholic liver disease. Gut 65:830-839. DOI 10.1136/gutjnl-2015-310585

Mekhlafi MA, Ibrahim BM, and Rayyis LA. 2018. Abnormal admission kidney function predicts higher mortality in stroke patients. Neurosciences (Riyadh) 23:200-203. DOI 10.17712/nsj.2018.3.20170521

Raphaeli G, Mazighi M, Pereira VM, Turjman F, and Striefler J. 2015. State-of-the-art endovascular treatment of acute ischemic stroke. Adv Tech Stand Neurosurg 42:33-68. DOI 10.1007/978-3-319-09066-5_3

Roger VL, Go AS, Lloyd-Jones DM, Adams RJ, Berry JD, Brown TM, Carnethon MR, Dai S, de Simone G, Ford ES, Fox CS, Fullerton HJ, Gillespie C, Greenlund KJ, Hailpern SM, Heit JA, Ho PM, Howard VJ, Kissela BM, Kittner SJ, Lackland DT, Lichtman JH, Lisabeth LD, Makuc DM, Marcus GM, Marelli A, Matchar DB, McDermott MM, Meigs JB, Moy CS, Mozaffarian D, Mussolino ME, Nichol 
G, Paynter NP, Rosamond WD, Sorlie PD, Stafford RS, Turan TN, Turner MB, Wong ND, WylieRosett J, American Heart Association Statistics C, and Stroke Statistics S. 2011. Heart disease and stroke statistics--2011 update: a report from the American Heart Association. Circulation 123:e18-e209. DOI 10.1161/CIR.0b013e3182009701

Schaubeck M, Clavel T, Calasan J, Lagkouvardos I, Haange SB, Jehmlich N, Basic M, Dupont A, Hornef M, von Bergen M, Bleich A, and Haller D. 2016. Dysbiotic gut microbiota causes transmissible Crohn's disease-like ileitis independent of failure in antimicrobial defence. Gut 65:225-237. DOI 10.1136/gutjnl2015-309333

Scher JU, Sczesnak A, Longman RS, Segata N, Ubeda C, Bielski C, Rostron T, Cerundolo V, Pamer EG, Abramson SB, Huttenhower C, and Littman DR. 2013. Expansion of intestinal Prevotella copri correlates with enhanced susceptibility to arthritis. Elife 2. ARTN e01202 DOI 10.7554/eLife.01202

Singh V, Roth S, Llovera G, Sadler R, Garzetti D, Stecher B, Dichgans M, and Liesz A. 2016. Microbiota Dysbiosis Controls the Neuroinflammatory Response after Stroke. J Neurosci 36:7428-7440. DOI 10.1523/JNEUROSCI.1114-16.2016

Wu WK, Chen CC, Liu PY, Panyod S, Liao BY, Chen PC, Kao HL, Kuo HC, Kuo CH, Chiu THT, Chen RA, Chuang HL, Huang YT, Zou HB, Hsu CC, Chang TY, Lin CL, Ho CT, Yu HT, Sheen LY, and Wu MS. 2018. Identification of TMAO-producer phenotype and host-diet-gut dysbiosis by carnitine challenge test in human and germ-free mice. Gut. DOI 10.1136/gutjnl-2018-317155

Yin J, Liao SX, He Y, Wang S, Xia GH, Liu FT, Zhu JJ, You C, Chen Q, Zhou L, Pan SY, and Zhou HW. 2015. Dysbiosis of Gut Microbiota With Reduced Trimethylamine-N-Oxide Level in Patients With Large-Artery Atherosclerotic Stroke or Transient Ischemic Attack. J Am Heart Assoc 4. DOI 10.1161/JAHA.115.002699

Zhang L, Li N, Cao K, Yang XY, Zeng G, Sun X, and He QY. 2017. Crucial residue Trp158 of lipoprotein PiaA stabilizes the ferrichrome-PiaA complex in Streptococcus pneumoniae. J Inorg Biochem 167:150-156. DOI 10.1016/j.jinorgbio.2016.08.015

Zheng P, Zeng B, Zhou C, Liu M, Fang Z, Xu X, Zeng L, Chen J, Fan S, Du X, Zhang X, Yang D, Yang Y, Meng H, Li W, Melgiri ND, Licinio J, Wei H, and Xie P. 2016. Gut microbiome remodeling induces depressive-like behaviors through a pathway mediated by the host's metabolism. Molecular Psychiatry 21:786-796. DOI 10.1038/mp.2016.44

Zhou HW, Li DF, Tam NFY, Jiang XT, Zhang H, Sheng HF, Qin J, Liu X, and Zou F. 2011. BIPES, a costeffective high-throughput method for assessing microbial diversity. Isme Journal 5:741-749. DOI 10.1038/ismej. 2010.160 
Figure 1

\section{Comparison ofa-diversity between the gut microbiota of patientsand controls.}

A: OTU Based PLS-DA Analysis. Orange triangles represent samples (intestinal microbiota) from patients; blue circles represent samples of controls. Five indices were used to represent the a-diversity between the patients and controls ( B: observed species, C: chao, D: ace, E: s hannon's diversity, F: s impson diversity) ; $\mathrm{G}$ : The number of community species between the patients and controls. $\mathrm{N}$ indicates healthy people and $\mathrm{P}$ indicates patients.

A

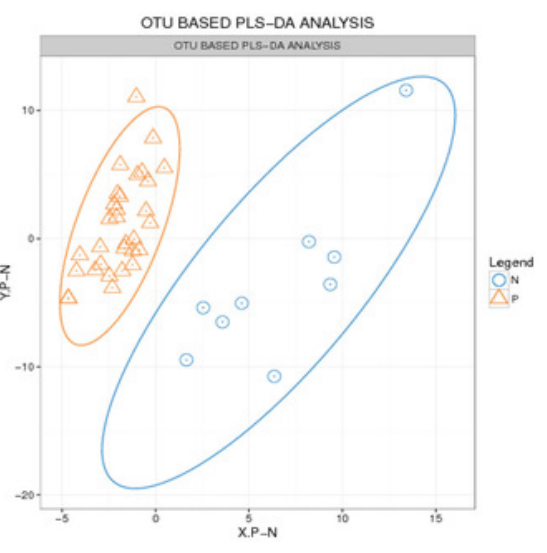

B

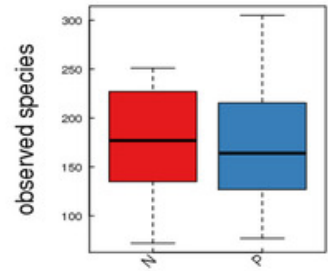

E

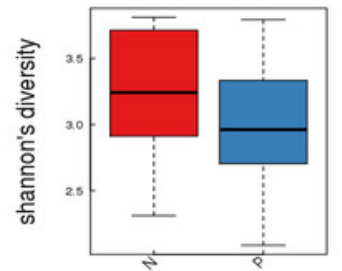

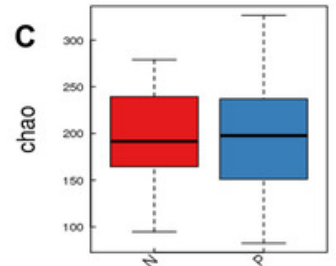
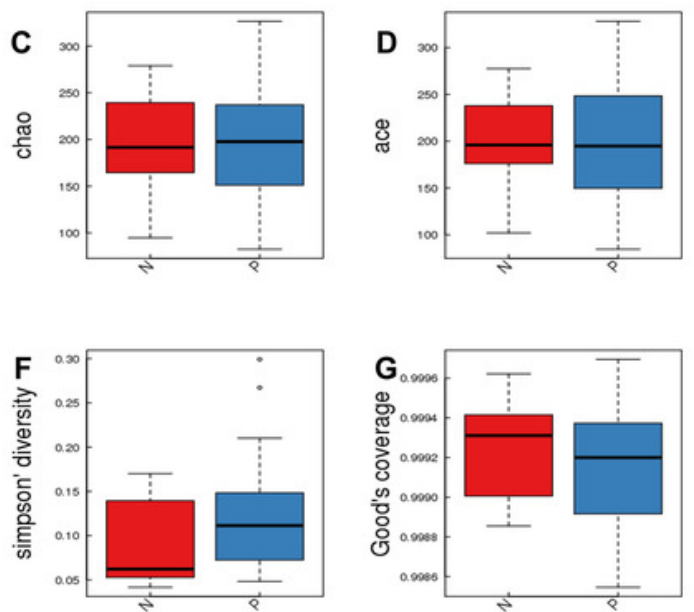


\section{Figure 2}

\section{Analysis of the variation of intestinal gut in species level incontrols and Acute Cerebral Infarction patients}

A: The relative abundance of each species in the intestinal gut in each sample from Acute Cerebral Infarction patients and controls ( $B$ indicates healthy people and A indicates patients ); The average relative abundance of three gut microbiota ( B: Blautia boeum, C: Streptococcus infantis, D: Prevotella copri) between patient group and control group. $\mathrm{P}<0.05$ was considered statistically significant in the comparison groups , $\mathrm{N}$ indicates normal people and $\mathrm{P}$ indicates patients. All the above analyses are $\mathrm{T}$-test.

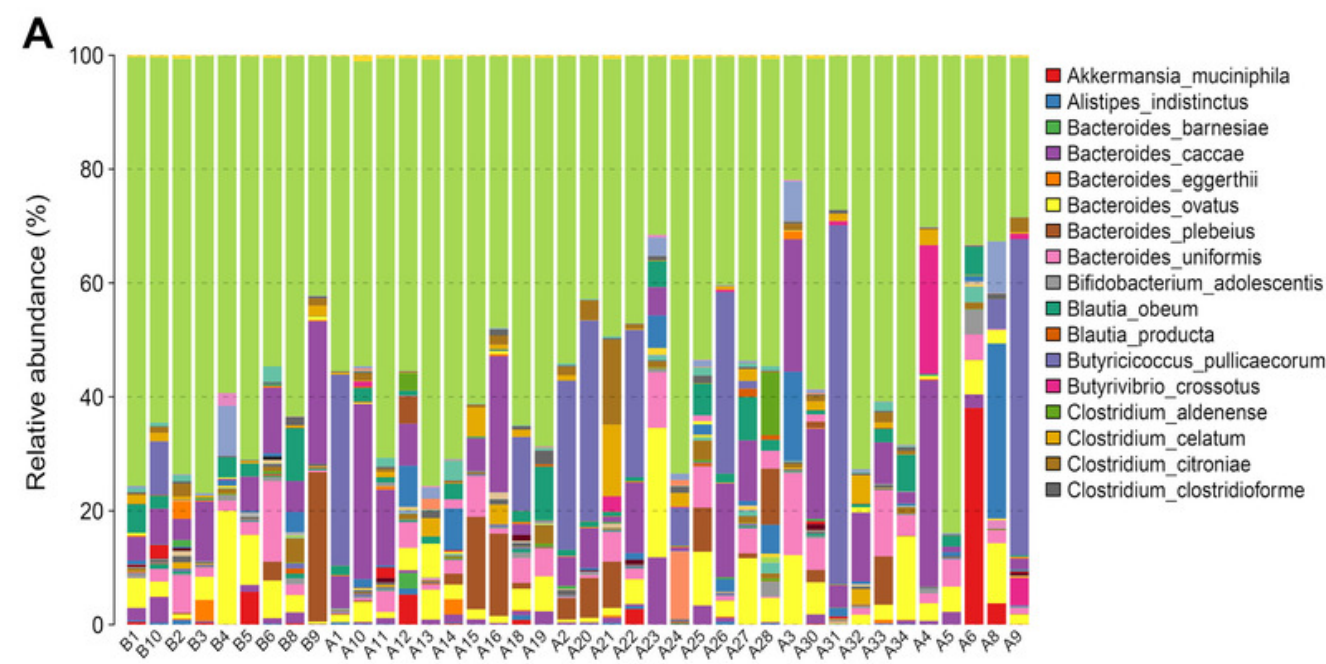

$\begin{array}{ll}\square \text { Clostridium_hathewayi } & \square \text { Lactobacillus_se } \\ \square \text { Clostridium_hiranonis } & \square \text { Oscillospira_guil } \\ \square \text { Clostridium_lavalense } & \square \text { Parabacteroides } \\ \square \text { Clostridium_perfringens } & \square \text { Parabacteroides } \\ \square \text { Clostridium_ramosum } & \square \text { Prevotella_copri } \\ \square \text { Clostridium_symbiosum } & \square \text { Prevotella_sterc } \\ \square \text { Collinsella_aerofaciens } & \square \text { Pyramidobacter } \\ \square \text { Collinsella_stercoris } & \square \text { Roseburia_faeci } \\ \square \text { Coprococcus_catus } & \square \text { Ruminococcus_ } \\ \square \text { Coprococcus_eutactus } & \square \text { Ruminococcus_ } \\ \square \text { Desulfovibrio_D168 } & \square \text { Ruminococcus } \\ \square \text { Escherichia_coli } & \square \text { Streptococcus_i } \\ \square \text { Eubacterium_biforme } & \square \text { Veillonella_dispe } \\ \square \text { Faecalibacterium_prausnitzii } & \square \text { Veillonella_parvi } \\ \square \text { Gemmiger_formicilis } & \square \text { Unclassified } \\ \square \text { Haemophilus_parainfluenzae } & \square \text { Others(<0.5\%) } \\ \square \text { Lactobacillus_mucosae } & \end{array}$

B

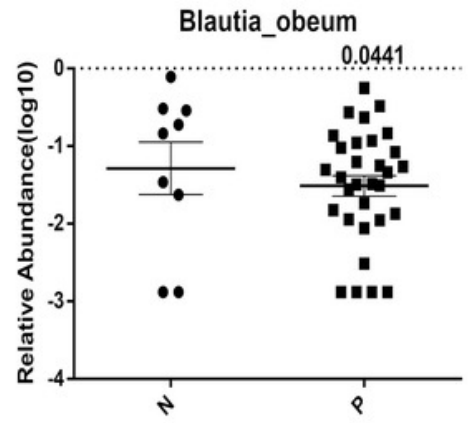

C

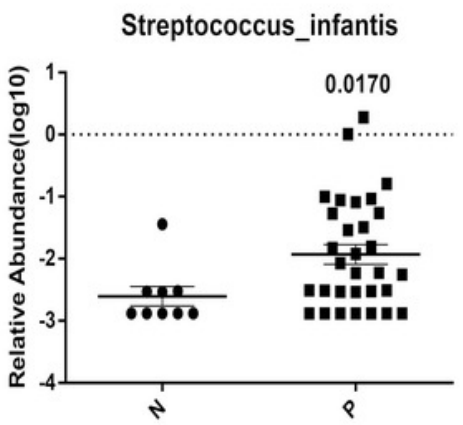

D

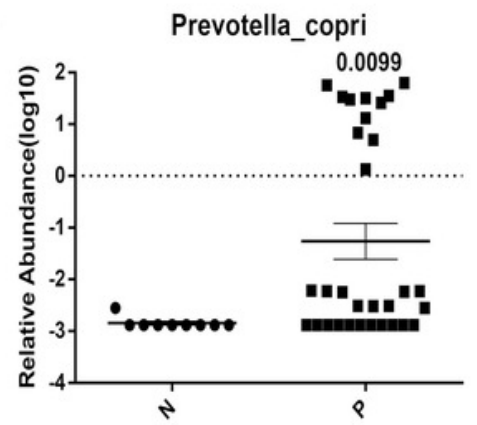


Figure 3

\section{Pathway abundance analysis of microbial taxa with KEGG .}

A: KEGG functional pathway abundance ana lysis of the gut microbiota (B indicates healthy people and $A$ indicates patients ); Average pathway abundance of a gut bacterium(B:Methane_metabolism, C: Lipopolysaccharide_biosynthesis_proteins, D: Secretion_system; E: Flagellar_assembly; F: Lipopolysaccharide_biosynthesis) between patient group and control group. All the above analyses are Ttest.

A
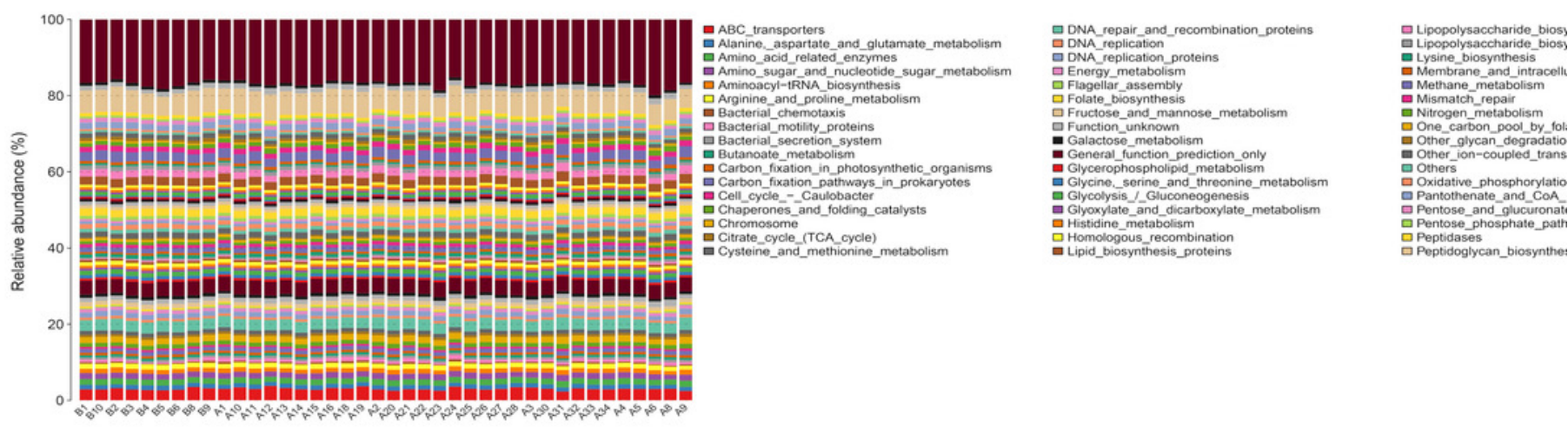

B

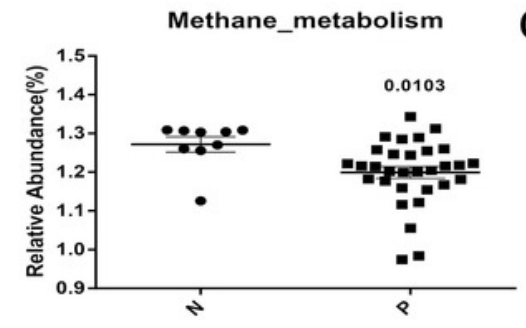

$E$

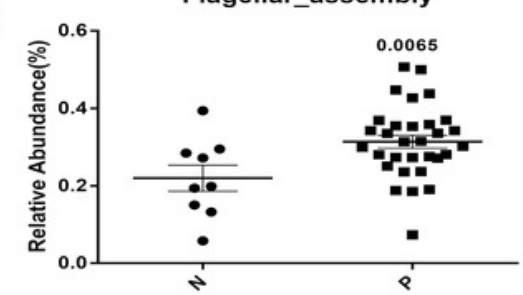

C

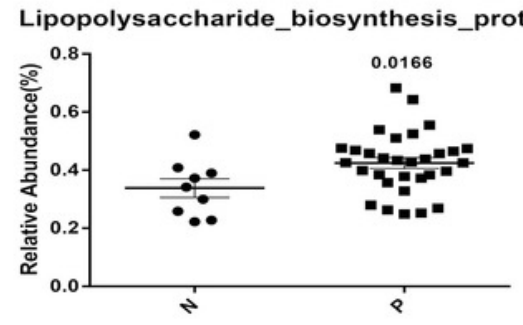

$\mathbf{F}$

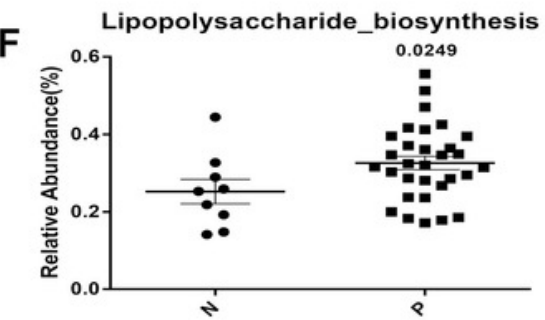

Secretion_system

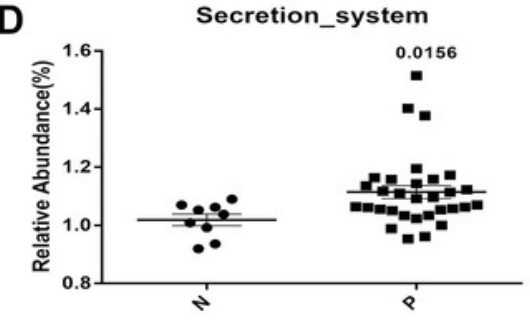




\section{Table $\mathbf{1}$ (on next page)}

Characteristics of the study participants

CAD, coronary artery disease; HBP, high blood pressure;HLP, hyperlipidemia; LAA, largeartery atherosclerosis; Continuous variables are presented as medians (interquartile range). Significant differences between groups were analyzed with the: $a$, Chi-square test; $b$ Kruskal Wallis Test. 
1 Table 1. Characteristics of the study participants

2

\begin{tabular}{llll}
\hline Variable & Patients & Controls & P Value \\
\hline $\mathrm{N}$ & 31 & 9 & - \\
Male, $\mathrm{n}(\%)$ & $22(70.97)$ & $6(66.67)$ & $0.385^{\mathrm{a}}$ \\
Age (Median, IQR) & $61(40-94)$ & $61(53-69)$ & $.238^{\mathrm{b}}$ \\
Current smoker(\%) & $19(61.29)$ & 0 & $0.001^{\mathrm{a}}$ \\
Previous diabetes, $\mathrm{n}(\%)$ & 0 & 0 & - \\
Previous CAD, $\mathrm{n}(\%)$ & 0 & 0 & - \\
Previous HBP, $\mathrm{n}(\%)$ & $23(74.19)$ & $2(22.22)$ & $0.005^{\mathrm{a}}$ \\
Previous HLP, $\mathrm{n}(\%)$ & $11(35.48)$ & 0 & $0.036^{\mathrm{a}}$ \\
Previous ischemic stroke(\%) & $1(3.23)$ & 0 & $0.585^{\mathrm{a}}$ \\
\hline
\end{tabular}




\section{Table 2 (on next page)}

Blood Biochemistry Results

LDL, low-density lipoprotein; TC, total cholesterol; TG, triglycerides; GLU $\square$ glucose; WBC, white blood cell count; $\mathrm{Cr}$, creatinine; UA, uric acid; LP(a),Lipoprotein(a). Continuous variables are presented as means \pm standard deviations or as medians (interquartile range).Significant differences between groups were analyzed with the: $a$, Kruskal Wallis Test; $b$, Student's $t$ test. 
1

2

3

Table2.Blood Biochemistry Results

\begin{tabular}{llll}
\hline Variable & Patients & Controls & P Value \\
\hline $\mathrm{TC}, \mathrm{mmol} / \mathrm{L}$ & $4.984 \pm 1.669$ & $4.089 \pm 0.624$ & $0.028^{\mathrm{a}}$ \\
$\mathrm{HDL}, \mathrm{mmol} / \mathrm{L}$ & $1.056 \pm 0.268$ & $1.437 \pm 0.268$ & $0.002^{\mathrm{a}}$ \\
$\mathrm{LDL}, \mathrm{mmol} / \mathrm{L}$ & $3.199 \pm 1.068$ & $2.712 \pm 0.548$ & $0.199^{\mathrm{a}}$ \\
$\mathrm{TG}, \mathrm{mmol} / \mathrm{L}$ & $1.93 \pm 1.239$ & $0.961 \pm 0.268$ & $0.003^{\mathrm{b}}$ \\
$\mathrm{GLU}, \mathrm{mmol} / \mathrm{L}$ & $5.132 \pm 0.645$ & $4.646 \pm 0.590$ & $0.178^{\mathrm{a}}$ \\
$\mathrm{WBC}, 10^{\wedge} \mathrm{L} / \mathrm{L}$ & $6.733 \pm 1.733$ & $4.932 \pm 1.456$ & $0.023^{\mathrm{a}}$ \\
$\mathrm{Cr}, \mu \mathrm{mol} / \mathrm{L}$ & $75.28 \pm 18.70$ & $59.52 \pm 8.443$ & $0.13^{\mathrm{a}}$ \\
$\mathrm{UA}, \mu \mathrm{mol} / \mathrm{L}$ & $329.4 \pm 93.95$ & $277.9 \pm 66.79$ & $0.134^{\mathrm{b}}$ \\
$\mathrm{LP}(\mathrm{a}), \mathrm{mg} / \mathrm{L}$ & $325.8 \pm 262.9$ & $74.89 \pm 38.72$ & $0.003^{\mathrm{c}}$ \\
\hline
\end{tabular}




\section{Table 3(on next page)}

Correlation between gut microbial taxa at the Species level and disease risk factors

P-values and $r^{2}$-values for linear regression. A + or - indicates positive or negative association, respectively. N.S indicates not significant. 
1 Table 3. Correlation between gut microbial taxa at the Species level and disease risk factors

\begin{tabular}{|c|c|c|c|c|c|c|c|c|c|}
\hline & $\mathrm{TC}$ & HDL & LDL & TG & GLU & WBC & $\mathrm{Cr}$ & UA & $\mathrm{LP}(\mathrm{a})$ \\
\hline Blautia & N.S & N.S & N.S & N.S & N.S & $\begin{array}{l}\mathrm{P}=0.04, \mathrm{r}^{2}= \\
0.1053 \text { - }\end{array}$ & N.S & N.S & N.S \\
\hline Prevotella & N.S & N.S & N.S & N.S & N.S & N.S & N.S & N.S & N.S \\
\hline Streptococcus & N.S & N.S & N.S & N.S & N.S & N.S & $\begin{array}{l}\mathrm{p}=0.022 \\
6, \mathrm{r}^{2}=0.1 \\
328+\end{array}$ & N.S & $\begin{array}{l}\mathrm{p}=0.0494, \\
\mathrm{r}^{2}=0.1004 \\
+\end{array}$ \\
\hline
\end{tabular}

2 